\title{
RACSAM
}

Rev. R. Acad. Cien. Serie A. Mat.

VOL. 103 (2), 2009, pp. 373-385

Matemática Aplicada / Applied Mathematics

\section{The price of liquidity in constant leverage strategies}

\author{
Marcos Escobar, Andreas Kiechle, Luis Seco and Rudi Zagst
}

\begin{abstract}
In this paper we develop a formula for the Liquidity Premium of constant leverage strategies (CLS). These financial products are path dependent options where the underlying typically is a hedge fund portfolio. We describe and explain the functionality of CLSs, showing a closed form expression for the price of a CLS on a hedge fund assuming a Geometric Brownian Motion, discrete rebalancing for the hedge fund investment as well as stochastic interest rates. The risk of default before the next rebalancing date leads to a liquidity premium for the CLS which increases with the volatility of the underlying hedge fund portfolio and the leverage of the strategy. An increasing rebalancing period first leads to a higher liquidity premium, however, as the rebalancing period is extended further the liquidity premium begins to shrink again.
\end{abstract}

\section{El precio de liquidez en estrategias con apalancamiento constante}

Resumen. La funcionalidad de las estrategias de apalancamiento constante (CLS) es investigada en este artículo. Estos productos financieros son opciones depedendientes del camino, donde los típicos subyacentes son Hedge Funds. En particular se encuentra una fórmula cerrada para el precio de liquidez de este derivado en el contexto de procesos brownianos geométricos con reajuste discreto de la cartera y tasa de interés estocástica. El riesgo de bancarrota antes de un reajuste conlleva a un precio de liquidez para el CLS, el cual es proporcional a la volatilidad del activo subyacente y al apalancamiento de la estrategia. Un incremento en el periodo entre reajustes implica un incremento inicial en el precio, sin embargo, el precio disminuye para largos periodos de reajuste.

\section{Introduction}

Constant Leverage Strategies are options where the underlying typically is a hedge fund portfolio. In this context leverage is defined as the ratio of debt to equity. An investment strategy with constant leverage works as follows. In addition to his own money, which we will refer to as the equity, the investor takes a loan and invests the sum of the equity and the loan in a portfolio. As the portfolio value changes over time, the leverage of the portfolio changes as well. The aim of a constant leverage strategy is to keep the initial leverage constant, which implies that the financing of the portfolio has to be adjusted. In the case that the value of the underlying portfolio has increased more than the interest on the loan, the investor has to take an additional loan to uphold the leverage. In the other case, the investor pays back a fraction of the loan. In the end, the investor pays back the cumulative loan (including interest) and his payoff is the value of the portfolio minus the loan and accrued interest. A CLS is a product where the issuer of the option sells a constant leverage strategy, typically on a hedge fund portfolio, to an investor. The underlying portfolio is

Presentado por / Submitted by Alejandro Balbás.

Recibido /Received: February 10, 2009. Aceptado /Accepted: April 1, 2009.

Palabras clave / Keywords: CPPI, hedge fund, liquidity premium, constant leverage strategy.

Mathematics Subject Classifications: 91B30, 47N30.

(C) 2009 Real Academia de Ciencias, España. 
financed by a loan which is provided by the issuer, and by a (large) fraction of the price of the CLS (i.e. the equity) which the issuer charges the investor. The remaining fraction of the price of the CLS is called the liquidity premium and is retained by the issuer.

The properties of continuous-time CLS strategies have been studied in the literature as particular cases of Constant proportion portfolio insurance (CPPI) ${ }^{1}$ strategies. Some clarifying papers on the topic of continuous CPPIs are Bookstaber and Langsam [8, (2000)], Black and Perold [7, (1992)] or Bertrand and Prigent [3, (2002)]. The literature on CPPI also deals with the effects of jump processes, stochastic volatility models and extreme value approaches on the CLS method, c.f. Bertrand and Prigent [4, (2002)], Bertrand and Prigent [5, (2003)]. Nevertheless the issue of discrete-time CLS has been barely covered; in a recent working paper Balder-Brandl-Mahayni [2, (2005)] analyze a discrete-time version of a general CPPI strategy which is used for risk management purposes. Therefore risk measure statistics like shortfall and expected shortfall given default are all computed under the real world measure.

One important feature of hedge funds is their illiquidity (see, e.g., Ineichen [10, (2002)]), which means that buying or selling shares of a hedge fund is restricted to specific dates. The period between two consecutive dates can range from one month to two years. Accordingly, the issuer can only change his investment in the underlying hedge fund portfolio of the CLS at these specific rebalancing dates. For the investor, the distinction between carrying out a constant leverage strategy by himself and buying a CLS arises in the case where the portfolio value falls under the loan between two consecutive rebalancing dates. As for the constant leverage strategy, this case corresponds to a negative equity which the investor owes to the issuer of the loan. Regarding the CLS we will refer to this case as the default of the CLS. The hedge fund portfolio is liquidated and the CLS expires worthless. The payoff for the investor is zero and the issuer takes the receipts to redeem the loan. Thus, the buyer of the CLS is in possession of a downside protection on his investment. While he could lose more than his original investment by carrying out a constant leverage strategy on his own, this is not possible if he buys the CLS. Here the option character of the CLS comes into existence for the investor. The price for this option or downside protection is the liquidity premium. Vice versa, the issuer of the CLS of course bears the risk of losing part of his loan as he cannot liquidate the hedge fund portfolio immediately when the portfolio value drops below the issued loan. Due to this liquidity risk, the issuer will charge more for the CLS than the initial value which he invests into the portfolio. The price of the CLS is composed by the money invested in the portfolio plus the liquidity premium accounting for the option the investor buys and which compensates the issuer for bearing the risk of default. In contrast to approaches which determine the liquidity premium for stocks using the expected illiquidity (see, e.g., Acharya and Lasse [1, (2003)]), the liquidity premium can be calculated in advance and is constant.

The three factors that influence the probability of default of the CLS and thus determine the liquidity premium are the volatility of the underlying portfolio, the length of the period between two rebalancing dates, and the leverage of the portfolio. Based on the descriptions above, a CLS can be seen as a string of call options with a maturity period identical to the timestep between two consecutive rebalancing dates. Obviously, the strike price corresponds to the loan plus accrued interest at a specific rebalancing date. As the leverage is reset to a constant level over time, the strike price of the option makes up a particular fraction of the current portfolio value. This means that the relation of portfolio value and strike price is constant at each rebalancing date, neglecting the case of default. On the other hand, the absolute value of the strike price, or the loan respectively, changes with the duration of the CLS. In this paper we focus on a discretetime CLS, and we price this strategy under the risk neutral measure obtaining a closed form expression for the liquidity premium.

This paper proceeds as follows. In Section 2 we define the CLS mathematically. In Section 3 we calculate the payoff of the CLS at maturity and derive the price of a CLS assuming a Geometric Brownian Motion for the underlying portfolio. To get a better understanding of the sensitivity of the CLS to market parameters, we elaborate its dynamic behavior in Section 4. Finally, we summarize our findings and draw a conclusion.

\footnotetext{
${ }^{1} \mathrm{~A}$ CLS could be seen as a CPPI with zero floor and multiplier equal to a function of the wanted leverage
} 


\section{Mathematical Definition of the CLS}

In this section we define the constant leverage strategy and CLS mathematically and demonstrate its functionality by giving a one-step example from one rebalancing date to the consecutive rebalancing date. All stochastic processes are defined on a filtered probability space $\left(\Omega, F,\left(F_{t}\right)_{t \in[0, T]}, P\right)$ which satisfies the standard hypotheses. We assume that the underlying $S$ of the CLS follows a Geometric Brownian Motion in the risk-neutral world where interest rates are stochastic ${ }^{2}$ and the short rate is $r_{t}$. Furthermore, we assume that the market is complete ${ }^{3}$, i.e. that there exists an equivalent martingale measure (see, e.g., Zagst [11, (2002)]) and that the dynamics of the stock, $S$ is:

$$
\mathrm{d} S_{t}=r_{t} S_{t} \mathrm{~d} t+\sigma S_{t} \mathrm{~d} W_{t}
$$

where $W_{t}$ is the standard Brownian Motion under the risk-neutral measure (see, e.g. Hull [9, (2005)]). Let $\alpha_{t}$ denote the amount of $S_{t}$ held from $t$ to $t+1$ so that the total money invested in the hedge fund portfolio in $t$ is $\alpha_{t} S_{t}$. Let $B_{t}^{a}$ be the value of the loan in $t$, or respectively the money borrowed from the riskless account, after rebalancing in $t$. $B_{t}^{a}$ grows to $B_{t+1}^{b}=\mathrm{e}^{\int_{t}^{t+1}} r(s) \mathrm{d} s B_{t}^{a}$ before the rebalancing in $t+1$ takes place. The equity $V_{t}$ in $t$ is the difference between the value of the hedge fund portfolio and the loan, $V_{t}=\alpha_{t} S_{t}-B_{t}^{a}$. Note that the process for $V_{t}$ is self-financing whereas the process for $\alpha_{t} S_{t}$ is not. The maturity of the CLS and thus the constant leverage strategy is $T$. Rebalancing occurs in $t_{i}, i=1,2, \ldots$, $N-1$, with $N:=T / \Delta t$ and $\Delta t$ is the timestep between two rebalancing dates.

To illustrate the functionality of the CLS, we give an one-step example from $t_{i}$ to $t_{i+1}$. After rebalancing in $t_{i}$ the portfolio value of the underlying hedge fund portfolio is $\alpha_{t_{i}} S_{t_{i}}$. This amount is financed by the equity $V_{t_{i}}$ and the loan $B_{t_{i}}^{a}$ where $B_{t_{i}}^{a}$ is chosen in such a way that the ratio of $B_{t_{i}}^{a}$ and $V_{t_{i}}$ equals the target leverage, i.e. the leverage of the portfolio is $L=B_{t_{i}}^{a} / V_{t_{i}}$. In $t_{i+1}$ the value of the portfolio before rebalancing is $\alpha_{t_{i}} S_{t_{i+1}}$, the new loan before rebalancing is

$$
B_{t_{i+1}}^{b}=\mathrm{e}^{\int_{t_{i}}^{t_{i+1}} r(s) \mathrm{d} s} B_{t_{i}}^{a}
$$

and the equity is $V_{t_{i+1}}=\alpha_{t_{i}} S_{t_{i+1}}-B_{t_{i+1}}^{b}$. Depending on the performance of $S$ we now have to distinguish between two cases:

Case $1 \alpha_{t_{i}} S_{t_{i+1}} \leq B_{t_{i+1}}^{b}$

In this case the portfolio value has dropped below the loan. As the equity $V_{t_{i+1}}$ is zero or negative in $t_{i+1}$ the CLS defaults and expires worthless. The owner of the option will get back nothing, i.e. his payoff is zero. The issuer of the option loses the difference of $B_{t_{i+1}}^{b}-\alpha_{t_{i}} S_{t_{i+1}}$.

Case $2 \alpha_{t_{i}} S_{t_{i+1}}>B_{t_{i+1}}^{b}$

In this case it is very likely that the leverage has changed and thus the financing of the portfolio has to be adjusted. The rebalancing of the portfolio occurs according to two conditions, the constant leverage condition and the self-financing condition for $V_{t_{i+1}}$. The constant leverage condition implies that it must hold

$$
B_{t_{i+1}}^{a}=L \cdot V_{t_{i+1}}=L\left(\alpha_{t_{i+1}} S_{t_{i+1}}-B_{t_{i+1}}^{a}\right) .
$$

Solving this for $B_{t_{i+1}}^{a}$ leads to

$$
B_{t_{i+1}}^{a}=\alpha_{t_{i+1}} S_{t_{i+1}} \cdot \frac{L}{1+L}, \quad \text { i.e. } \quad \alpha_{t_{i+1}} S_{t_{i+1}}=(1+L) V_{t_{i+1}}
$$

\footnotetext{
${ }^{2}$ The calculations will show that the particular process for the interest rate has no influence in the value of the CLS.

${ }^{3}$ The hedge fund is a derivative in the market not an underlying, and the market itself is arbitrage free and complete. The assets of the hedge fund, are traded continuously in the market and the rebalancing of the hedge fund is discrete.
} 
Thus, the amount of money invested in the underlying portfolio is a constant multiple of the equity. Here we can see the similarity to the CPPI strategy (see e.g. Black and Jones [6, (1987)]). Due to the self-financing condition for $V_{t}$ it must also hold that

$$
V_{t_{i+1}}=\alpha_{t_{i}} S_{t_{i+1}}-B_{t_{i+1}}^{b}=\alpha_{t_{i+1}} S_{t_{i+1}}-B_{t_{i+1}}^{a} .
$$

By solving (3) for $\alpha_{t_{i+1}}$ we obtain

$$
\begin{aligned}
& \alpha_{t_{i+1}}=\frac{V_{t_{i+1}}+B_{t_{i+1}}^{a}}{S_{t_{i+1}}}=\frac{V_{t_{i+1}}+L V_{t_{i+1}}}{S_{t_{i+1}}} \\
& =(1+L) \frac{V_{t_{i+1}}}{S_{t_{i+1}}}=(1+L) \frac{\left(\alpha_{t_{i}} S_{t_{i+1}}-B_{t_{i+1}}^{b}\right)}{S_{t_{i+1}}} \quad \text { last equality by (3) } \\
& =(1+L) \frac{\left(\alpha_{t_{i}} S_{t_{i+1}}-\mathrm{e}^{\int_{t_{i}}^{t_{i+1}} r(s) \mathrm{d} s} B_{t_{i}}^{a}\right)}{S_{t_{i+1}}} \\
& =(1+L) \frac{\alpha_{t_{i}}\left(S_{t_{i+1}}-S_{t_{i}} \frac{L}{1+L} \mathrm{e}^{\int_{t_{i}}^{t_{i+1}} r(s) \mathrm{d} s}\right)}{S_{t_{i+1}}} \\
& =\alpha_{t_{i}}(1+L)\left(1-\frac{L}{1+L} \frac{S_{t_{i}}}{S_{t_{i+1}}} \mathrm{e}^{\int_{t_{i}}^{t_{i+1}} r(s) \mathrm{d} s}\right) \\
& =\alpha_{t_{i}}+\alpha_{t_{i}}\left(1-\frac{S_{t_{i}}}{S_{t_{i+1}}} \mathrm{e}^{\int_{t_{i}}^{t_{i+1}} r(s) \mathrm{d} s}\right) L .
\end{aligned}
$$

Thus we get

$$
\left(1-\frac{S_{t_{i}}}{S_{t_{i+1}}} \mathrm{e}^{\int_{t_{i}}^{t_{i+1}} r(s) \mathrm{d} s}\right)<0 \quad \text { if } \quad \frac{S_{t_{i+1}}}{S_{t_{i}}}<\mathrm{e}^{\int_{t_{i}}^{t_{i+1}} r(s) \mathrm{d} s}
$$

and

$$
\left(1-\frac{S_{t_{i}}}{S_{t_{i+1}}} \mathrm{e}^{\int_{t_{i}}^{t_{i+1}} r(s) \mathrm{d} s}\right)>0 \quad \text { if } \quad \frac{S_{t_{i+1}}}{S_{t_{i}}}>\mathrm{e}^{\int_{t_{i}}^{t_{i+1}} r(s) \mathrm{d} s},
$$

where $S_{t_{i+1}} / S_{t_{i}}$ denotes the performance of the hedge fund portfolio and $\mathrm{e}^{\int_{t_{i}}^{t_{i+1}} r(s) \mathrm{d} s}$ is the bond performance in $\left[t_{i}, t_{i+1}\right]$. Hence,

$$
\begin{array}{ll}
\alpha_{t_{i+1}}<\alpha_{t_{i}} & \text { in Case 1 and } \\
\alpha_{t_{i+1}}>\alpha_{t_{i}} & \text { in Case 2. }
\end{array}
$$

Using Equation (3) we thus get

$$
\begin{aligned}
& B_{t_{i+1}}^{a}=\left(\alpha_{t_{i+1}}-\alpha_{t_{i}}\right) S_{t_{i+1}}+B_{t_{i+1}}^{b}<B_{t_{i+1}}^{b} \\
& \text { in Case } 1 \text { and } \\
& B_{t_{i+1}}^{a}=\left(\alpha_{t_{i+1}}-\alpha_{t_{i}}\right) S_{t_{i+1}}+B_{t_{i+1}}^{b}>B_{t_{i+1}}^{b} \\
& \text { in Case } 2 .
\end{aligned}
$$

We can draw the following conclusion. In Case 1 we get

$$
\alpha_{t_{i+1}}<\alpha_{t_{i}} \quad \text { and } \quad B_{t_{i+1}}^{a}<B_{t_{i+1}}^{b},
$$

which means that the loan is decreased to match the initial leverage of the portfolio. For Case 2 we get

$$
\alpha_{t_{i+1}}>\alpha_{t_{i}} \quad \text { and } \quad B_{t_{i+1}}^{a}>B_{t_{i+1}}^{b},
$$


which corresponds to an increase of the loan to match the initial leverage. By substituting the result for $\alpha_{t_{i+1}}$ from Equation (4) into (2), we get for $B_{t_{i+1}}^{a}$

$$
\begin{aligned}
B_{t_{i+1}}^{a} & =\alpha_{t_{i}}(1+L)\left(1-\frac{L}{1+L} \frac{S_{t_{i}}}{S_{t_{i+1}}} \mathrm{e}^{\int_{t_{i}}^{t_{i+1}} r(s) \mathrm{d} s}\right) S_{t_{i+1}} \frac{L}{1+L} \\
& =\alpha_{t_{i}} L\left(1-\frac{L}{1+L} \frac{S_{t_{i}}}{S_{t_{i+1}}} \mathrm{e}^{\int_{t_{i}}^{t_{i+1}} r(s) \mathrm{d} s}\right) S_{t_{i+1}} .
\end{aligned}
$$

With this we can now state the new portfolio after rebalancing in $t_{i+1}$

$$
\alpha_{t_{i+1}} S_{t_{i+1}}=V_{t_{i+1}}+B_{t_{i+1}}^{a}
$$

where $\alpha_{t_{i+1}}$ and $B_{t_{i+1}}^{a}$ are given by Equations (4) and (5).

Bringing the two cases together, we can now give the value of the equity for the investor in the CLS after one step from $t_{i}$ to $t_{i+1}$.

$$
\begin{aligned}
V_{t_{i+1}} & = \begin{cases}\alpha_{t_{i}} S_{t_{i+1}}-B_{t_{i+1}}^{b} & \text { if } \alpha_{t_{i}} S_{t_{i+1}}>B_{t_{i+1}}^{b} \\
0 & \text { if } \alpha_{t_{i}} S_{t_{i+1}} \leq B_{t_{i+1}}^{b}\end{cases} \\
& =\max \left\{\alpha_{t_{i}} S_{t_{i+1}}-B_{t_{i+1}}^{b}, 0\right\}
\end{aligned}
$$

The value of the loan for the issuer after one step from $t_{i}$ to $t_{i+1}$ is

$$
B_{t_{i+1}}^{b}=\min \left\{\mathrm{e}^{\int_{t_{i}}^{t_{i+1}} r(s) \mathrm{d} s} B_{t_{i}}^{a}, \alpha_{t_{i}} S_{t_{i+1}}\right\}
$$

This equation for $B_{t_{i+1}}^{b}$ clearly shows the risk of the bank to lose part of its loan. Above we argued that due to this risk the bank will charge a liquidity premium. Hence, the price of the CLS: CO $\left(t_{0}\right)$ is

$$
\mathrm{CO}\left(t_{0}\right)=V_{t_{0}}+(\text { Liquidity Premium })
$$

The liquidity premium arises because of the illiquidity of hedge funds. In the case of continuous returns and liquidity of the underlying of the CLS, the liquidity premium disappears and the price of the CLS is $V_{t_{0}}$. In this case there is no reason for the issuer to charge a premium as the loan doesn't bear any default risk because the hedge fund portfolio can be liquidated as soon as the portfolio value approaches the value of the loan.

As we can see from the previous example, the Constant Leverage strategy is a pro-cyclical strategy. In the case that the underlying portfolio has returned more than the interest on the loan from $t$ to $t+1$, the equity makes up a bigger fraction of the portfolio at the next rebalancing $t+1$, or in other words, the leverage of the portfolio has decreased. Therefore, an additional loan has to be taken in $t+1$ to set up the portfolio with the initial leverage. On the other hand, in the case the underlying portfolio has returned less than the interest on the loan (but has not defaulted), the leverage of the portfolio has increased and thus some of the loan will be paid back to restore the initial leverage.

\section{Liquidity Premium and the price of CLS.}

With the setting from above we can first calculate the price of a CLS and then the value of the liquidity premium. This price in $t_{0}=0$ is composed by the equity $V_{0}$ plus the liquidity premium which compensates the bank for the risk of losing part of its loan. We first derive the payoff of the CLS at maturity and afterwards calculate the price of the CLS as the discounted expected value of the payoff at maturity. In order to do this, recall that the CLS can be seen as a string of single call options, each with a maturity of $\Delta t$. Thus, we have to check at each rebalancing date if the CLS has defaulted or not since the last time 
we have rebalanced. This means that the payoff at maturity is conditional on the fact that the option has not defaulted before. To represent the conditional payoff we use the Indicator Function. This function only takes two values, 1 for a certain set of events, and 0 for the complementary set of events:

$$
\mathbf{1}_{A}(x)= \begin{cases}1 & \text { if } x \in A \\ 0 & \text { if } x \notin A\end{cases}
$$

Let us now calculate the payoff of the CLS at maturity $T$. We transform this payoff and express it in terms of a normally distributed random variable. This transformation will facilitate the calculation of the expectated payoff in the following.

Proposition 1 Let $Y_{t_{i}}:=W_{t_{i}}-W_{t_{i-1}}$ and $z:=\left(\ln \frac{L}{1+L}+\frac{\sigma^{2}}{2} \Delta t\right) / \sigma$. Then the payoff of a CLS at maturity time $T=t_{N}$ is given by

$$
\mathrm{CO}(T)=(1+L)^{N} V_{t_{0}} \mathrm{e}^{\int_{t_{0}}^{t_{N}} r(s) \mathrm{d} s} \cdot \prod_{i=1}^{N}\left(\mathrm{e}^{-\frac{\sigma^{2}}{2} \Delta t+\sigma Y_{t_{i}}}-\frac{L}{1+L}\right) \cdot \prod_{i=1}^{N} 1_{\left\{Y_{t_{i}}>z\right\}} .
$$

Proof. See Appendix A.

With this result we can now calculate the price of the CLS in $t_{0}$ by calculating the discounted expectation of the payoff derived in the above proposition (see, e.g., Zagst [11, (2002)]).

Proposition 2 The price of the CLS specified by the settings above is

$$
\mathrm{CO}\left(t_{0}\right)=(1+L)^{N} V_{t_{0}} \cdot\left(N\left(d_{1}\right)-\frac{L}{1+L} N\left(d_{2}\right)\right)^{N},
$$

where $d_{1}$ and $d_{2}$ are given by

$$
d_{1}=\frac{\ln \frac{1+L}{L}+\frac{\sigma^{2}}{2} \Delta t}{\sigma \sqrt{\Delta t}}, \quad d_{2}=d_{1}-\sigma \sqrt{\Delta t} .
$$

Proof. See Appendix A.

Note that in particular the price of the CLS in (8) is independent of the stochastic interest rate $r_{t}$.

Proposition 3 The price of the $C L S, \mathrm{CO}\left(t_{0}\right)$, can be written as

$$
\mathrm{CO}\left(t_{0}\right)=V_{t_{0}} \cdot\left[1+(1+L) \cdot \text { Call }\left(\frac{L}{1+L}, 1,0, \sigma, \Delta t\right)\right]^{N},
$$

where Call $(S, X, r, \sigma, t)$ denotes the Black-Scholes call price with underlying price $S$, exercise price $X$, riskless return $r$, volatility $\sigma$, and time to maturity $t$.

Proof. See Appendix A.

The value of the Liquidity cost is provided next as a result of Equation (6) and Proposition 3.

Corollary 1 The value of the Liquidity Premium, $\mathrm{LI}\left(t_{0}\right)$, can be written as:

$$
\mathrm{LI}\left(t_{0}\right)=V_{t_{0}} \cdot\left(\left[1+(1+L) \cdot \operatorname{Call}\left(\frac{L}{1+L}, 1,0, \sigma, \Delta t\right)\right]^{N}-1\right),
$$

where Call $(S, X, r, \sigma, t)$ is as before. 


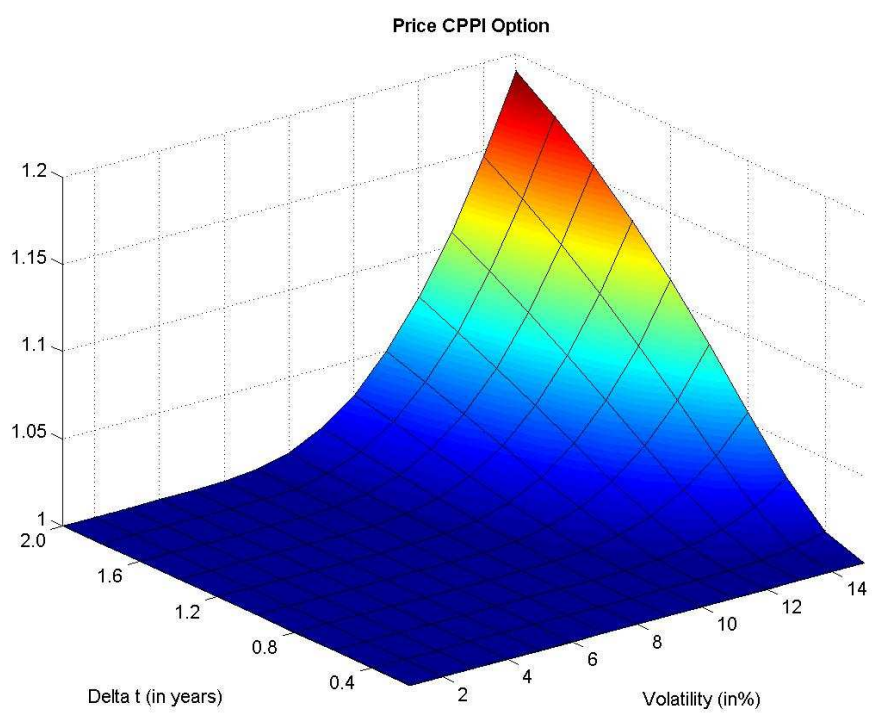

Figure 1. Price of the CPPI Option, $T=10$ years, $L=3, V_{t_{0}}=1.0$

\section{Dynamic Behaviour of Liquidity Premium}

For a deeper understanding of the CLS we now show how the price of the CLS which we have deduced in the previous section depends on the volatility $\sigma$ of the underlying hedge fund portfolio, the period between two consecutive rebalancing dates $\Delta t$ and the leverage $L$ of the portfolio. First, recall that the price of the CLS is composed by the initial investment of the investor plus the liquidity premium due to the illiquidity of a hedge fund investment. This liquidity premium is strongly related to the probability of default of the CLS. For the investor the option character of the CLS comes into existence in the case of default. The three factors that influence the probability of default of the CLS and thus determine the liquidity premium are $\sigma$, $\Delta t$, and $L$. As we have showed above, the price of the CLS is independent of the stochastic interest rate $r_{t}$.

Figures 1 and 2 show the price of the CLS in $t_{0}$ with a maturity of $T=10$ years depending on $\sigma$ and $\Delta t$ with a leverage of $L=3$ (Figure 1) and a leverage of $L=5$ (Figure 2). The initial equity $V_{t_{0}}$ is one. We chose values up to $15 \%$ for $\sigma$ and up to 2 years for $\Delta t$ which are typical for a hedge fund portfolio.

As we can see in both figures, the price of the CLS is one, which is the initial investment of the investor, for small values of $\sigma$ and $\Delta t$. This means that the liquidity premium is close to zero. The reason for this is that in these cases almost no defaults occur. This becomes clear if we recall that default happens when the underlying portfolio loses more than the value of the equity from one rebalancing date to the next one. Thus, default is unlikely if the portfolio exhibits a low standard deviation and is rebalanced frequently.

Figures 1 and 2 show that the liquidity premium increases with inreasing values for $\sigma$ and $\Delta t$. As the volatility of the underlying portfolio increases and the distance between two consecutive rebalancing dates gets bigger the probability of default is rising as well. In the case of default, the buyer of the CLS is better off than with a direct investment in the underlying portfolio and therefore has to pay for the liquidity premium of the CLS. Figure 2 shows that the liquidity premium can make up for a large fraction of the price of the CLS. For a leverage $L=5, \sigma=15 \%$ and $\Delta t=2$ years for example the liquidity premium accounts for 0.7 of the price of the option which means that the CLS is almost twice as expensive as a direct investment. The reason for this high liquidity premium is that in this case the probability of default for the CLS during the maturity is $72 \%$. Furthermore, we can see that higher values for the leverage $L$ lead to a higher liquidity premium. This is obvious as the equity represents a smaller value of a highly leveraged 


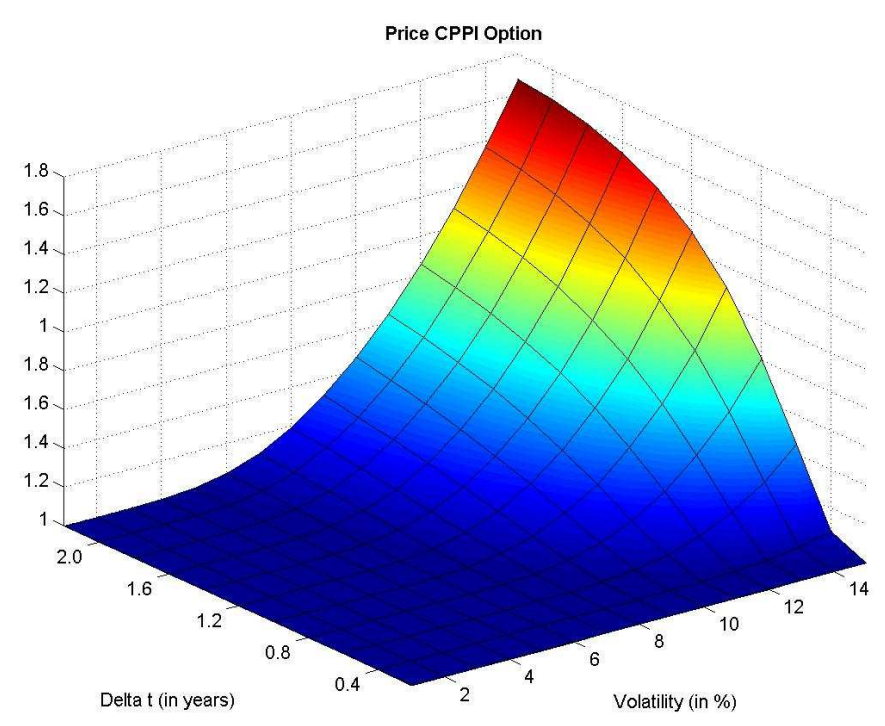

Figure 2. Price of the CPPI Option, $T=10$ years, $L=5, V_{t_{0}}=1.0$

portfolio and thus smaller losses of the underlying portfolio can result in default.

In Figures 1 and 2 the behaviour of the price of the CLS is quite intuitive and easy to understand. To give a complete picture, we extend the volatility for the underlying portfolio to a maximum of $40 \%$ in Figure 3. At the first glance the fact that the option price is falling again with increasing $\Delta t$ for greater values of $\sigma$ may be surprising and counter-intuitive.

To understand this behaviour of the price, we have to have a closer look at the occurrence of defaults. Figure 4 shows the probability of default for a CLS with $T=10$ years and $L=3$ depending on $\sigma$ and $\Delta t$. It illustrates that the probability of default first increases and then decreases with growing $\Delta t$. Recall that with continuous rebalancing there are no defaults. If one waits longer for the next rebalancing, e.g. one or three months, the probability of default increases. With a rebalancing period of one year the probability for this option to default peaks at about $80 \%$. However, the surprising conclusion of this plot is that if the rebalancing period is increased further, the probability of default is decreasing again.This behaviour can be explained as follows. Imagine two (not very realistic) CLSs with the same maturity $T=10$ years, one rebalancing once after five years $(\mathrm{CO} 1)$, the other one with no rebalancing at all (CO 2). Let the other parameters for this example be $\sigma=25 \%$ and $L=3$. The probability of default for $\mathrm{CO} 1$ is higher than for $\mathrm{CO} 2$ simply because there are two possibilities for it to default (in $t=5$ years and in $t=10$ years) compared to only one in $t=10$ years for CO 2. Or in mathematical terms, the probability of default for CO 1 after five years is $41 \%$. The overall probability of default for CO 1 thus is $41 \%+(100 \%-41 \%) \cdot 41 \%=65 \%$. The probability for a default of CO 2 after ten years is $51 \%$. Hence the probability of default is greater if we conduct a rebalancing after five years compared to the case where we do not rebalance at all. Knowing that the price of the CLS is closely related to the number of defaults we can now understand why the price of the CLS begins to decrease again for large values of $\Delta t$ in Figure 3. The reason for this behaviour of the price is that the probability of default is beginning to decrease for large $\Delta t$.

However, these theoretical examinations may not be very relevant in practice. Looking at the maximum of the probability of default in the previous example we can reason that nobody would use a product that has such high probabilities of default to invest in hedge funds. For a reasonable range of the probability of default (e.g. up to $5 \%$ or 10\%) the price of the CLS increases with increasing $\sigma$ and $\Delta t$ as it is shown in Figure 1. 


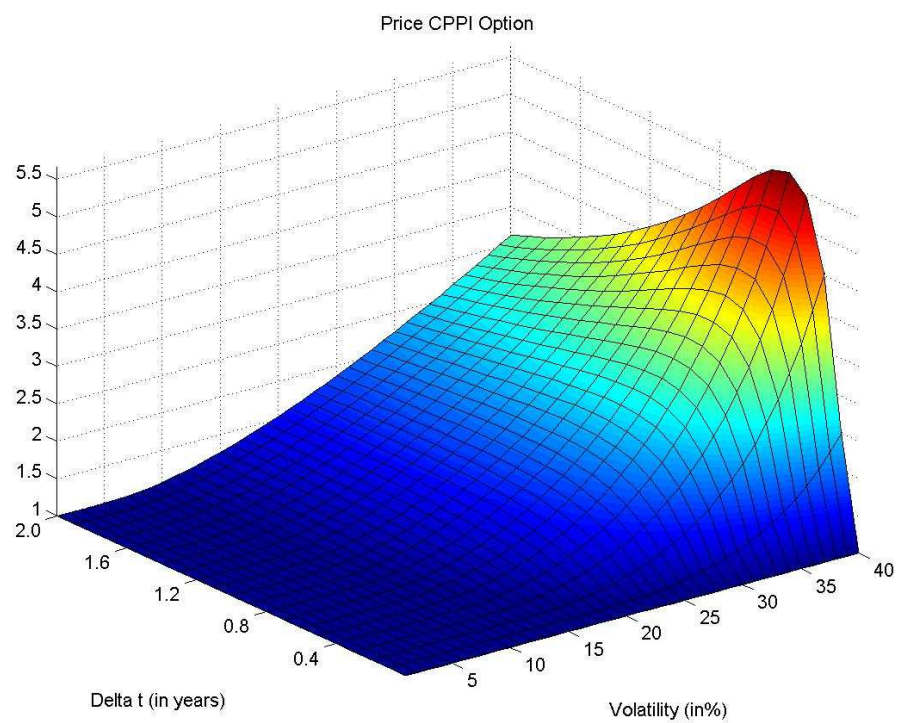

Figure 3. Price of the CPPI Option, $T=10$ years, $L=3, V_{t_{0}}=1.0$

\section{Summary and Conclusion}

In this paper we calculated the value of the liquidity premium that appears as a result of discrete rebalancing of Constant Leverage Strategies. The main assumptions are a Geometric Brownian Motion for the underlying hedge fund portfolio and stochastic interest rates. Due to the fact that hedge funds can be traded only at specific dates, the CLS can default between two rebalancing dates and thus the price of the CLS must contain a liquidity premium. The three parameters which influence this premium are the volatility $\sigma$ of the underlying portfolio, the timestep $\Delta t$ between two consecutive rebalancing dates and the leverage $L$ which determines the financing of the underlying portfolio. We found out that greater values for $\sigma$ and the $L$ lead to a greater liquidity premium and thus a higher price of the CLS. The reason for this is that with increasing $\sigma$ and $L$ the probability of default of the CLS also increases. The influence of $\Delta t$ on the option price depends on the specific combination of all three factors $\sigma, \Delta t$ and $L$. We discovered that for higher but fixed values of $\sigma$ and $L$ the impact of $\Delta t$ on the probability of default changes with increasing $\Delta t$ and leads to a hump in the function of the price of the CLS. Finally, it turned out that the price of the CLS does not depend on the stocastic interest rate $r_{t}$.

\section{References}

[1] Acharya, V. And Lasse, H. P., (2003). Asset Pricing and Liquidity Risk, London Business School, working paper.

[2] Balder, Sven, Brandl, Michael And Mahayni, Antje, (2005). Effectiveness of CPPI Strategies under Discrete-Time Trading, working paper.

[3] Bertrand, P. And Prigent, J.-L., (2002). Portfolio Insurance Strategies: OBPI versus CPPI, discussion paper, GREQAM and Universite Montpellier1.

[4] Bertrand, P. And Prigent, J.-L., (2002). Portfolio Insurance: The Extreme Value Approach to the CPPI, Finance, 23, 68-86. 


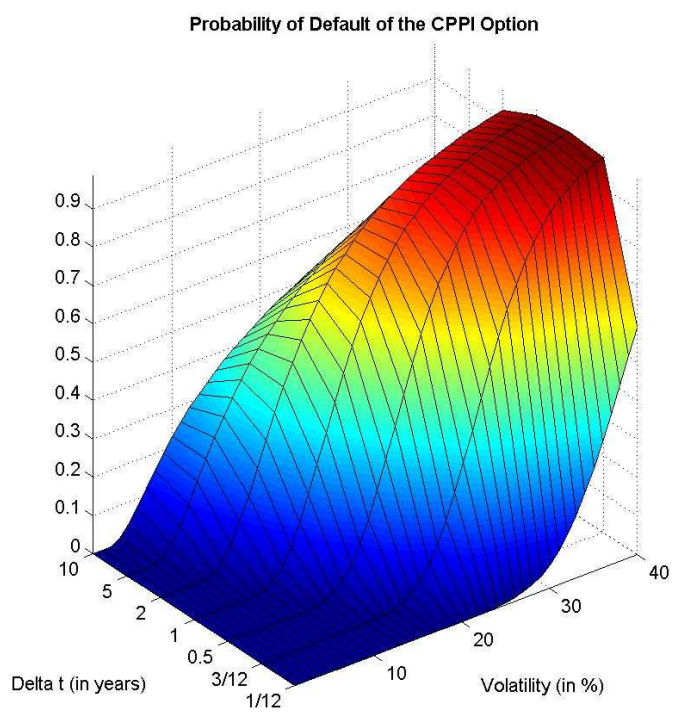

Figure 4. Probalility of default, $T=10$ years, $L=3$

[5] Bertrand, P. And Prigent, J.-L., (2003). Portfolio Insurance Strategies: A Comparison of Standard Methods When the Volatility of the Stoch is Stochastic, International Journal of Business, 8, 15-31.

[6] Black, F. And Jones, R., (1987). Simplifying portfolio insurance, The Journal of Portfolio Management, 14, 1, 48-51.

[7] Black, F. And Perold, A. R., (1992). Theory of constant proportion portfolio insurance, J. Econ. Dynamics Control, 16, 403-426.

[8] Bookstaber, R. And Langsam, J. A., (2000). Portfolio Insurance Trading Rules (Digest Summary), Journal of Futures Markets, 20, 1, 41-57.

[9] Hull, J. C., (2005). Options, Futures and Other Derivatives, Pearson Prentice Hall.

[10] InEICHEn, A. M., (2002). Absolute Returns: The Risk and Opportunities of Hedge Fund Investing, Wiley Finance.

[11] ZAGST, R., (2002). Interest Rate Management, Springer Finance.

\section{A Appendix}

ProOf OF PROPOSITION 1. For the following calculation of the payoff of the CLS at maturity $T$ we first need two results:

1. Let $X_{t_{i}}:=S_{t_{i-1}} / S_{t_{i}}$, then

$$
\prod_{s=1}^{N-1} X_{t_{s}}=\frac{S_{t_{0}}}{S_{t_{N-1}}}, \quad \text { i.e. } \quad S_{t_{N-1}}=\frac{S_{t_{0}}}{\prod_{i=1}^{t_{N}-1} X_{t_{i}}}
$$


2. Recall that $S_{t_{i}}=S_{0} \mathrm{e}^{\int_{0}^{t_{i}} r(s) \mathrm{d} s-\frac{\sigma^{2}}{2} t_{i}+\sigma W_{t_{i}}}$. Then,

$$
X_{t_{i}}=\frac{S_{t_{i-1}}}{S_{t_{i}}}=\mathrm{e}^{-\int_{t_{i-1}}^{t_{i}} r(s) \mathrm{d} s+\frac{\sigma^{2}}{2} \Delta t-\sigma Y_{t_{i}}}
$$

Recall that $W_{t} \sim N(0, t)$, which implies that $Y_{t_{i}} \sim N(0, \Delta t)$. Note that in particular $Y_{t_{i}}$ and $Y_{t_{j}}$ are independent for $i, j=1, \ldots, N$ and $i \neq j$.

Now we can give the payoff of the CLS at maturity $T=t_{N}$. The payoff in $T$ is the excess of the portfolio value in $T$ over the loan $B_{T}^{b}$ on the condition that there has been no default in $t_{i}, i=1, \ldots, N$. As the $Y_{t_{i}}$ are independent, we can simply multiply the probabilities of default to obtain the conditional payoff in $T$. Furthermore, due to the constant leverage property, these probabilities of default are the same for each $t_{i}, i=1, \ldots, N$.

The payoff of the CLS at maturity $T=t_{N}$ on the condition that no default has occured in $t_{i}, i=1, \ldots$, $N-1$ is $\alpha_{t_{N-1}} S_{t_{N}}-B_{t_{N}}^{b}$. We now transform this payoff to express it in terms of $Y_{t}$ which is normally distributed.

$$
\mathrm{CO}(T)=\left(\alpha_{t_{N-1}} S_{t_{N}}-\mathrm{e}^{\int_{t_{N-1}}^{t_{N}} r(s) \mathrm{d} s} B_{t_{N-1}}^{a}\right)^{+} \cdot \prod_{i=1}^{N-1} 1\left\{\alpha_{t_{i-1}} S_{t_{i}}>\mathrm{e}^{\int_{t_{i-1}}^{t_{i}} r(s) \mathrm{d} s}{ }_{B_{t_{i-1}}^{a}}\right\}
$$

applying (2)

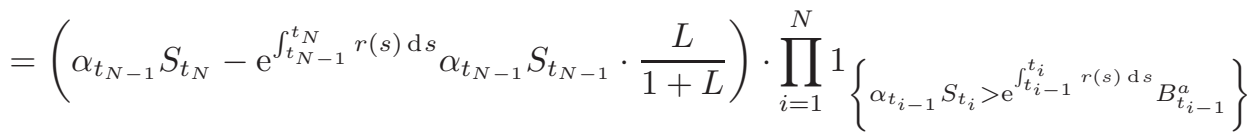

$$
\begin{aligned}
& =\alpha_{t_{N-1}} S_{t_{N-1}}\left(\frac{S_{t_{N}}}{S_{t_{N-1}}}-\mathrm{e}^{\int_{t_{N-1}}^{t_{N}} r(s) \mathrm{d} s} \frac{L}{1+L}\right) \cdot \prod_{i=1}^{N}\left\{\alpha_{t_{i-1}} S_{t_{i}}>\mathrm{e}^{\int_{t_{i-1}}^{t_{i}} r(s) \mathrm{d} s} B_{t_{i-1}}^{a}\right\}
\end{aligned}
$$

aplying (4)

$$
\begin{aligned}
&=\alpha_{t_{0}} \cdot \prod_{i=1}^{N-1}\left((1+L)\left(1-\frac{L}{1+L} \mathrm{e}^{\int_{t_{i-1}}^{t_{i}} r(s) \mathrm{d} s} \frac{S_{t_{i-1}}}{S_{t_{i}}}\right)\right) S_{t_{N-1}} \\
& \cdot\left(\frac{S_{t_{N}}}{S_{t_{N-1}}}-\mathrm{e}^{\int_{t_{N-1}}^{t_{N}} r(s) \mathrm{d} s} \frac{L}{1+L}\right) \cdot \prod_{i=1}^{N}\left\{\alpha_{t_{i-1}} S_{t_{i}}>\mathrm{e}^{\int_{t_{i-1}}^{t_{i}} r(s) \mathrm{d} s} B_{t_{i-1}}^{a}\right\} \\
&=\alpha_{t_{0}}(1+L)^{N-1} S_{t_{N-1}} \cdot \prod_{i=1}^{N-1}\left(1-\frac{L}{1+L} \mathrm{e}^{\int_{t_{i-1}}^{t_{i}} r(s) \mathrm{d} s} \frac{S_{t_{i-1}}}{S_{t_{i}}}\right) \\
& \cdot\left(\frac{S_{t_{N}}}{S_{t_{N-1}}}-\mathrm{e}^{\int_{t_{N-1}}^{t_{N}} r(s) \mathrm{d} s} \frac{L}{1+L}\right) \cdot \prod_{i=1}^{N}\left\{\frac{S_{t_{i}}}{S_{t_{i-1}}} \frac{1+L}{L}>\mathrm{e}^{\int_{t_{i-1}}^{t_{i}} r(s) \mathrm{d} s}\right\}
\end{aligned}
$$

applying (9)

$$
\begin{array}{r}
=\alpha_{t_{0}}(1+L)^{N-1} \frac{S_{t_{0}}}{\prod_{i=1}^{N-1} X_{t_{i}}} \cdot \prod_{i=1}^{N-1}\left(1-\frac{L}{1+L} \mathrm{e}^{\int_{t_{i}-1}^{t_{i}} r(s) \mathrm{d} s} X_{t_{i}}\right) \\
\cdot\left(\frac{1}{X_{t_{N}}}-\mathrm{e}^{\int_{t_{N-1}}^{t_{N}} r(s) \mathrm{d} s} \frac{L}{1+L}\right) \prod_{i=1}^{N}\left\{X_{t_{i}<\frac{1+L}{L}} \mathrm{e}^{-\int_{t_{i-1}}^{t_{i}} r(s) \mathrm{d} s}\right\}
\end{array}
$$




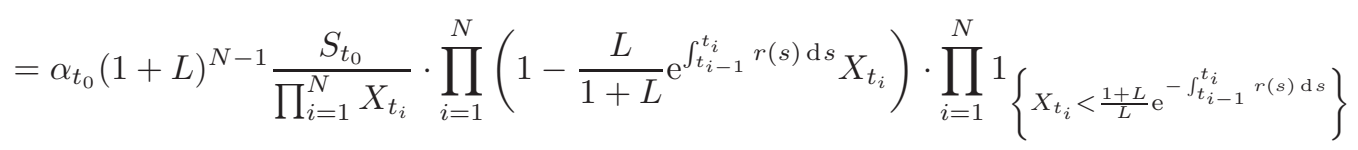

applying (9) again

$$
\begin{aligned}
= & \alpha_{t_{0}}(1+L)^{N-1} S_{t_{0}} \cdot \prod_{i=1}^{N}\left(\mathrm{e}^{\int_{t_{i}-1}^{t_{i}} r(s) \mathrm{d} s-\frac{\sigma^{2}}{2} \Delta t+\sigma Y_{t_{i}}}\right) \\
& \cdot \prod_{i=1}^{N}\left(1-\frac{L}{1+L} \mathrm{e}^{\int_{t_{i-1}}^{t_{i}} r(s) \mathrm{d} s} \mathrm{e}^{-\int_{t_{i-1}}^{t_{i}} r(s) \mathrm{d} s+\frac{\sigma^{2}}{2} \Delta t-\sigma Y_{t_{i}}}\right) \cdot \prod_{i=1}^{N}\left\{Y_{t_{i}>\frac{\ln }{1+L}+\frac{\sigma^{2}}{2} \Delta t}^{\sigma}=: z\right\} \\
= & \alpha_{t_{0}}(1+L)^{N-1} S_{t_{0}} \cdot \prod_{i=1}^{N}\left(\mathrm{e}^{\int_{t_{i-1}}^{t_{i}} r(s) \mathrm{d} s-\frac{\sigma^{2}}{2} \Delta t+\sigma Y_{t_{i}}}-\frac{L}{1+L} \mathrm{e}^{\int_{t_{i-1}}^{t_{i}} r(s) \mathrm{d} s}\right) \cdot \prod_{i=1}^{N} 1_{\left\{Y_{t_{i}}>z\right\}} \\
= & \alpha_{t_{0}}(1+L)^{N-1} S_{t_{0}} \mathrm{e}^{t_{t_{0}}^{t_{N}} r(s) \mathrm{d} s} \cdot \prod_{i=1}^{N}\left(\mathrm{e}^{-\frac{\sigma^{2}}{2} \Delta t+\sigma Y_{t_{i}}}-\frac{L}{1+L}\right) \cdot \prod_{i=1}^{N} 1_{\left\{Y_{t_{i}}>z\right\}}
\end{aligned}
$$

Now we know from Equation (2) that $\alpha_{t_{i+1}} S_{t_{i+1}}=(1+L) V_{t_{i+1}}$.

PROOF OF PROPOSITION 2. Let $z:=\left(\ln \frac{L}{1+L}+\frac{\sigma^{2}}{2} \Delta t\right) / \sigma$ and $f_{y_{t_{i}}}$ denote the density function of $Y_{t_{i}}$, i.e. the normal distribution $\mathrm{N}(0, \Delta t)$. Then the price of the CLS today is the expected value of the discounted payoff of the CLS in $T$.

$$
E_{t_{0}}[\mathrm{CO}(T)]=E\left[\mathrm{e}^{-\int_{t_{0}}^{T} r(s) \mathrm{d} s} \cdot \mathrm{CO}(T)\right]
$$

applying (7)

$$
\begin{aligned}
= & \alpha_{t_{0}}(1+L)^{N-1} S_{t_{0}} \int_{z}^{\infty} \cdots \int_{z}^{\infty} \prod_{i=1}^{N}\left(\mathrm{e}^{-\frac{\sigma^{2}}{2} \Delta t+\sigma y_{i}}-\frac{L}{1+L}\right) \cdot f\left(y_{1}\right) \cdots f\left(y_{2}\right) \mathrm{d} y_{1} \cdots \mathrm{d} y_{N} \\
= & \alpha_{t_{0}}(1+L)^{N-1} S_{t_{0}} \prod_{i=1}^{N} \int_{z}^{\infty}\left(\mathrm{e}^{-\frac{\sigma^{2}}{2} \Delta t+\sigma y_{i}}-\frac{L}{1+L}\right) \cdot\left(\frac{1}{\sqrt{2 \pi \Delta t}} \mathrm{e}^{-\frac{y_{i}^{2}}{2 \Delta t}}\right) \mathrm{d} y_{i} \\
= & \alpha_{t_{0}}(1+L)^{N-1} S_{t_{0}} \\
& \quad \prod_{i=1}^{N}\left(\int_{z}^{\infty} \frac{1}{\sqrt{2 \pi \Delta t}} \mathrm{e}^{-\frac{1}{2 \Delta t}\left(y_{i}^{2}-2 \sigma \Delta t y_{i}+\sigma^{2} \Delta t^{2}\right)} \mathrm{d} y_{i}-\frac{L}{1+L} \int_{z}^{\infty} \frac{1}{\sqrt{2 \pi \Delta t}} \mathrm{e}^{-\frac{y_{t_{i}}^{2}}{2 \Delta t}} \mathrm{~d} y_{t_{i}}\right) \\
= & \alpha_{t_{0}}(1+L)^{N-1} S_{t_{0}} \cdot \prod_{i=1}^{N}\left(\left(1-\mathrm{N}\left(\frac{z-\sigma \Delta t}{\sqrt{\Delta t}}\right)\right)-\frac{L}{1+L}\left(1-\mathrm{N}\left(\frac{z}{\sqrt{\Delta t}}\right)\right)\right) \\
= & \alpha_{t_{0}}(1+L)^{N-1} S_{t_{0}} \cdot\left(\left(1-\mathrm{N}\left(\frac{z-\sigma \Delta t}{\sqrt{\Delta t}}\right)\right)-\frac{L}{1+L}\left(1-\mathrm{N}\left(\frac{z}{\sqrt{\Delta t}}\right)\right)\right)
\end{aligned}
$$

Substituting $z$ leads to

$$
\mathrm{CO}\left(t_{0}\right)=\alpha_{t_{0}}(1+L)^{N-1} S_{t_{0}} \cdot\left(\mathrm{N}\left(\frac{\ln \frac{1+L}{L}+\frac{\sigma^{2}}{2} \Delta t}{\sigma \sqrt{\Delta t}}\right)-\frac{L}{1+L} \mathrm{~N}\left(\frac{\ln \frac{1+L}{L}-\frac{\sigma^{2}}{2} \Delta t}{\sigma \sqrt{\Delta t}}\right)\right)^{N}
$$


Proof of Proposition 3. Note that for the price CO $\left(t_{0}\right)$ of the CLS we get, using the put-call parity,

$$
\begin{aligned}
\mathrm{CO}_{1}\left(t_{0}\right)=(1+L)^{N} V_{t_{0}} \cdot\left[\begin{array}{c}
\mathrm{N}\left(-\frac{\ln \left(\frac{L}{1+L}\right)-\frac{\sigma^{2}}{2} \Delta t}{\sigma \sqrt{\Delta t}}\right) \\
-\frac{L}{1+L} \cdot \mathrm{N}\left(-\frac{\ln \left(\frac{L}{1+L}\right)+\frac{\sigma^{2}}{2} \Delta t}{\sigma \sqrt{\Delta t}}\right)
\end{array}\right]^{N} \\
=(1+L)^{N} V_{t_{0}} \cdot\left[\operatorname{Put}\left(\frac{L}{1+L}, 1,0, \sigma, \Delta t\right)\right]^{N} \\
=(1+L)^{N} V_{t_{0}} \cdot\left[1-\frac{L}{1+L}+\operatorname{Call}\left(\frac{L}{1+L}, 1,0, \sigma, \Delta t\right)\right]^{N} \\
=V_{t_{0}} \cdot\left[1+(1+L) \cdot \operatorname{Call}\left(\frac{L}{1+L}, 1,0, \sigma, \Delta t\right)\right]^{N} \cdot
\end{aligned}
$$

We therefore get a liquidity premium per rebalancing period $\Delta t$ of

$$
(1+L) \cdot \text { Call }\left(\frac{L}{1+L}, 1,0, \sigma, \Delta t\right)
$$

\section{Marcos Escobar}

Department for Mathematics,

Ryerson University,

Munich

escobareryerson. ca

\section{Luis Seco}

Director, Risklab Toronto, Sigma Analysis \& Management, Toronto

\section{Andreas Kiechle}

Munich University of Technology,

\section{Rudi Zagst}

Director, HVB-Institute for Mathematical Finance, Munich University of Technology,

Munich,

zagst@ma.tum.de 